Point for Consideration in Use of the Stereoscope 639

In conclusion I express my lasting gratitude to my late chiefs. To Mr. Foster Moore for the privilege I enjoyed of assisting him in his pioneer work on this subject, for the stimulus of his clear and honest observations, and for his generosity in allowing me to treat some of his cases at St. Bartholomew's Hospital. To Sir John Parsons, that " Master " of the physiology and pathology of the eye, I owe a high example of the manner in which to seek academic truth.

\title{
REFERENCES
}

Cade, Stanford.-Malignant Disease and its Treatment by Radium. John Wright and Sons Ltd., 1940.

Ellis, F.-Brit. Jl. Radiol., Vol XII, pp. 327-352, 1939.

MOORE, R. Foster.-Brit. Jl.Ophthal., Vol. XIV, p. 145, 1930; Trans. Ophthal. Soc. U.K., 1933.

Ninth Ann. Rep. Nat. Radium Commission, 1937-8.

Stallard, H. B.- Radiant Energy, Brit. Jl. Ophthal., Monograph Supplement, 1933; Brit. Med. Jl., Vol. II, p. 962, 1936; Brit. Jl. Ophthal., Vol. XXII, p. 604, 1938; Eye Surgery, John Wright and Sons Ltd., 1946.

WALDMAN N.-Arch. of Ophthal., Vol. XXIII, p. 55, 1940.

\section{A POINT FOR CONSIDERATION IN THE USE OF THE STEREOSCOPE}

BY

\author{
FranK W. LÁW \\ LONDON
}

IT recently occurred to the writer that it would be of value to know the answer to the question "What happens to the inclination of the visual axes on moving the stereogram in a stereoscope towards or away from the observer?'? The question seemed simple; the answer was not obvious. Further, enquiry in various directions discovered the somewhat consoling result that the answer was not generally known; indeed, it had not been considered by most of those asked, which is surprising, in view of the common habit of sending a patient home to "do exercises with a stereoscope."

On investigating the subject it is soon apparent that the interocular distance is a factor of fundamental importance. This can be demonstrated by considering three diagrams (Figs. 1, 2 and 3) in which the stereogram card is depicted as placed at three different distances from the lenses of the instrument. Fig. 1 shows it placed at the focal plane; the emergent pencil of light has parallel rays, and the amount of convergence is independent of the interocular distance. In Fig. 2 the card is represented as nearer than the focal plane; the emergent pencils are divergent and greater inter- 
ocular distance involves greater convergence. In Fig. 3, with the card beyond the focal plane, the emergent pencils are convergent, and greater interocular distance involves less convergence. In each figure the lens combinations are represented diagramatically; the optical centres are at 0.

One may now re-consider the phenomenon depicted in the figures by studying a chart obtained by calculation. Table I shows the

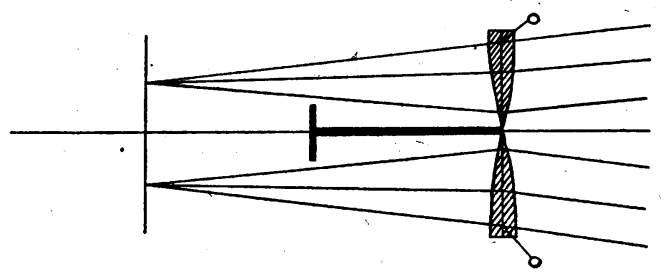

FIG. i.

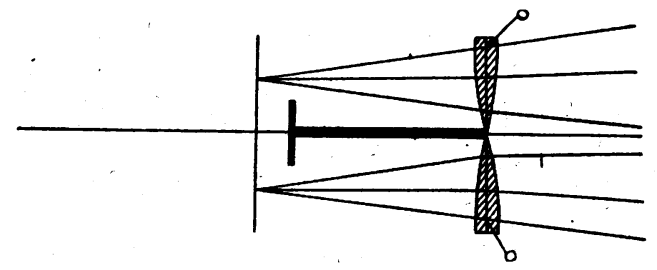

FIG. 2.

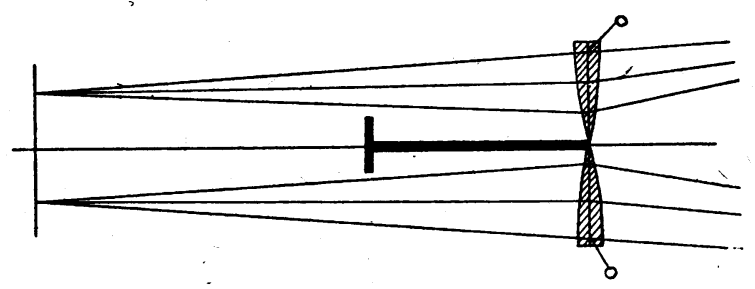

FIG. 3.

calculations for a stereoscope with lenses of +5.0D., optical centres $8.8 \mathrm{cms}$. apart, pictures separated by $5 \mathrm{cms}$. Three positions of the stereogram are considered. The fact, depicted in Fig 1, that when the card is placed at the focal plane, convergence is independent of interocular distance, emerges at once. Other interesting facts also appear; the same stereogram moved in the same direction and through the same distance induces an increase of convergence when the interocular distance is $5.2 \mathrm{cms}$, a smaller increase when it is $5.4 \mathrm{cms}$, and an actual decrease when 
it is $5.8 \mathrm{cms}$. Further, with the card at $25 \mathrm{cms}$., a greater interocular distance involves a reduced degree of convergence.'

Table II, calculated for a stereoscope with lenses of $+4.0 \mathrm{D}$., optical centres $9 \mathrm{cms}$. apart, and $5 \mathrm{cms}$. separation of the stereograms, shows lower convergence figures, but exactly the same tendencies.

TABLE I

\begin{tabular}{|c|c|c|c|c|c|}
\hline \multirow{2}{*}{$\begin{array}{c}\text { Width of } \\
\text { Stereo- } \\
\text { gram }\end{array}$} & \multirow{2}{*}{$\begin{array}{c}\text { Distance } \\
\text { from } \\
\text { Lenses }\end{array}$} & \multicolumn{4}{|c|}{ - Interocular Distance } \\
\hline & & $5.2 \mathrm{~cm}$ & $54 \mathrm{~cm}$ & $5.8 \mathrm{~cm}$. & $\mathrm{o}^{\top} 2 \mathrm{~cm}$ \\
\hline & & Conver & gence in & Prism & Dioptres \\
\hline $5 \mathrm{~cm}$. & $\begin{array}{l}10 \\
20 \\
25\end{array}$ & $\begin{array}{l}17.2 \\
19.0 \\
19.4\end{array}$ & $\begin{array}{l}18.1 \\
19.0 \\
19.2\end{array}$ & $\begin{array}{l}19.8 \\
19.0 \\
18.1\end{array}$ & $\begin{array}{l}21^{\circ} 6 \\
19.0 \\
183\end{array}$ \\
\hline
\end{tabular}

TABLE II

\begin{tabular}{|c|c|c|c|c|c|}
\hline \multirow{2}{*}{$\begin{array}{c}\text { Width of } \\
\text { Stereo- } \\
\text { gram }\end{array}$} & \multirow{2}{*}{$\begin{array}{l}\text { Distance } \\
\text { from } \\
\text { Lenses }\end{array}$} & \multicolumn{4}{|c|}{ Interocular Distance } \\
\hline & & $5.2 \mathrm{~cm}$ & $5.4 \mathrm{~cm}$ & $5.8 \mathrm{~cm}$. & $6.2 \mathrm{~cm}$ \\
\hline & & Conver & gence in & Prism & Dioptres \\
\hline $5 \mathrm{~cm}$ & $\begin{array}{l}11 \mathrm{~cm} . \\
25 \\
33.3\end{array}$ & $\begin{array}{l}14: 7 \\
16: 0 \\
16.3\end{array}$ & $\begin{array}{l}15^{\circ} 5 \\
16^{\circ} 0 \\
16^{\circ} 1\end{array}$ & $\begin{array}{l}16.3 \\
16.0 \\
15.7\end{array}$ & $\begin{array}{l}17 \cdot 1 \\
16.0 \\
15.3\end{array}$ \\
\hline
\end{tabular}

The practical deduction to be made from the above is that, once one has decided for what exact purpose one requires a patient to use a stereoscope, it is essential to choose an appropriate instrument and, having measured the patient's interocular distance, to calculate how the instrument will have to be used, and instruct the patient accordingly, With this in view, tables have been constructed showing the actual deviation of the visual axes involved for varying width of stereogram, and varying positions of the pictures. In Table III the basis is for lenses of $5.0 \mathrm{D}$., optical centres $8.8 \mathrm{cms}$. apart; in Table IV the lenses are 4.0D. in strength, with optical centres $9 \mathrm{cms}$. apart. In each case the distance from lens to ocular centre is assumed to be $3 \mathrm{cms}$. ; + (plus) indicates convergence and - (minus) divergence.

The heavy stepped line which crosses each Table is an important line of demarcation. It will be seen that the figures above this line demonstrate a common tendency-that an approach of the stereograms towards the eyes causes an increase of convergence; those below the line, that an approach of the stereograms towards 
the eyes causes a decrease in convergence, or an increase in divergence.

The practical application of these Tables would be relatively simple. Having ascertained the patient's interocular distance one can see at a glance which card and which direction of movement will give the effect desired in the particular case to be treated. This will at least prevent the patient from being asked to do exercises inappropriate to his condition-and one cannot but feel that such an event must have occurred. It may, however, be considered that, when convergence-or divergence is the only factor which it is desired to vary, a simpler course would be to give the patient a stereoscope with rotating prisms and place the stereograms in the focal plane.

TABLE III

\begin{tabular}{|c|c|c|c|c|c|c|c|c|c|}
\hline \multicolumn{2}{|c|}{ Stereogram } & \multicolumn{7}{|c|}{ Interocular Distance $(\mathrm{cm})}$. & \multirow{2}{*}{$\frac{-}{66}$} \\
\hline Width & & 6 & 4.8 & $5 \cdot 0$ & 522 & 54 & 58 & 6.2 & \\
\hline $3 \mathrm{~cm}$. & $\begin{array}{l}10 \mathrm{~cm} . \\
20 \\
25\end{array}$ & $\begin{array}{l}+32.2 \\
+29 \\
+28.3\end{array}$ & $\begin{array}{l}33 \cdot 1 \\
29 \\
28 \cdot 1\end{array}$ & $\begin{array}{l}34 \\
29 \\
27 \cdot 9\end{array}$ & $\begin{array}{l}34 \cdot 9 \\
29 \\
27 \cdot 7\end{array}$ & $\begin{array}{r}358 \\
29 \\
-275\end{array}$ & $\begin{array}{l}37 \cdot 6 \\
29 \\
27 \cdot 1\end{array}$ & $\begin{array}{l}39 \cdot 4 \\
29 \\
26 \cdot 7\end{array}$ & $\begin{array}{l}41 \cdot 2 \\
29 \\
26 \cdot 3\end{array}$ \\
\hline $4 \mathrm{~cm}$ & $\begin{array}{l}10 \mathrm{~cm} . \\
20 \\
25\end{array}$ & $\begin{array}{l}+23.4 \\
+24 \\
+24.1\end{array}$ & $\begin{array}{l}24 \cdot 3 \\
24 \\
23 \cdot 9\end{array}$ & $\begin{array}{l}25 \cdot 2 \\
24 \\
23 \cdot 7\end{array}$ & $\begin{array}{l}26 \cdot 1 \\
24 \\
23 \cdot 5\end{array}$ & $\begin{array}{l}27 \\
24 \\
23 \cdot 3\end{array}$ & $\begin{array}{l}28 \cdot 8 \\
24 \\
22 \cdot 9\end{array}$ & $\begin{array}{l}30 \cdot 6 \\
24 \\
22 \cdot 5\end{array}$ & $\begin{array}{l}32 \cdot 4 \\
24 \\
22 \cdot 1\end{array}$ \\
\hline $5 \mathrm{~cm}$ & $\begin{array}{l}10 \mathrm{~cm} . \\
20 \\
25\end{array}$ & $\begin{array}{l}+146 \\
+19 \\
+20\end{array}$ & $\begin{array}{l}15 \cdot 5 \\
19 \\
19 \cdot 8\end{array}$ & $\begin{array}{l}16^{\circ} 4 \\
19 \\
19^{\circ} 6\end{array}$ & $\begin{array}{l}17 \cdot 2 \\
19 \\
19 \cdot 4\end{array}$ & $\begin{array}{l}18^{\circ} 1 \\
19 \\
19 \cdot 2\end{array}$ & $\begin{array}{l}19 \cdot 8 \\
19 \\
18 \cdot 7\end{array}$ & $\begin{array}{l}21 \cdot 6 \\
19 \\
183 \\
\end{array}$ & $\begin{array}{l}23.4 \\
19 \\
17.9\end{array}$ \\
\hline $6 \mathrm{~cm}$. & $\begin{array}{l}10 \mathrm{~cm} . \\
20 \\
25\end{array}$ & $\begin{array}{l}+5.8 \\
+14 \\
+15.9\end{array}$ & $\begin{array}{l}6 \cdot 7 \\
14 \\
15 \cdot 7\end{array}$ & $\begin{array}{c}76 \\
14 \\
15.5\end{array}$ & $\begin{array}{l}8 \cdot 4 \\
14 \\
15 \cdot 3\end{array}$ & $\begin{array}{l}9 \cdot 3 \\
14 \\
15 \cdot 0\end{array}$ & $\begin{array}{l}11 \\
14 \\
146\end{array}$ & $\begin{array}{l}12 \cdot 8 \\
14 \\
14 \cdot 2\end{array}$ & $\begin{array}{l}14^{\circ} 6 \\
14 \\
13 \cdot 8 \\
\end{array}$ \\
\hline $7 \mathrm{~cm}$. & $\begin{array}{l}10 \mathrm{~cm} . \\
20 \\
25\end{array}$ & $\begin{array}{l}-2.9 \\
+\quad 9 \\
+118\end{array}$ & $\begin{array}{l}-2 \\
+\quad 9 \\
+116\end{array}$ & $\begin{array}{c}-11 \\
+\quad 9 \\
+11.4\end{array}$ & $\begin{array}{l}-0.2 \\
+\quad 9 \\
+11.2\end{array}$ & $\begin{array}{l}+\quad 0.6 \\
+\quad 9 \\
+11\end{array}$ & $\begin{array}{l}+\quad 2.3 \\
+\quad 9 \\
+\quad 10.5\end{array}$ & $\begin{array}{l}+41 \\
+\quad 9 \\
+10.1\end{array}$ & $\begin{array}{l}+\quad 58 \\
+\quad 9 \\
+\quad 9.7\end{array}$ \\
\hline $8 \mathrm{~cm}$. & $\begin{array}{l}10 \mathrm{~cm} . \\
20 \\
25\end{array}$ & $\begin{array}{l}-11.4 \\
+\quad 4 . \\
+\quad 7.6\end{array}$ & $\begin{array}{l}-10.5 \\
+\quad 4 \\
+\quad 74\end{array}$ & $\begin{array}{l}-9.6 \\
+\quad 4 \\
+\quad 7.2\end{array}$ & $\begin{array}{l}-8.7 \\
+\quad 4 \\
+\quad 7\end{array}$ & $\begin{aligned}- & 79 \\
+ & 4 \\
+ & 68\end{aligned}$ & $\begin{array}{l}-6.2 \\
+\quad 4 \\
+\quad 6.4\end{array}$ & $\begin{array}{l}-4.4 \\
+\quad 4 \\
+\quad 6\end{array}$ & $\begin{array}{l}-27 \\
+\quad 4 \\
+\quad 56\end{array}$ \\
\hline $9 \mathrm{~cm}$. & $\begin{array}{l}10 \mathrm{~cm} . \\
20 \\
25\end{array}$ & $\begin{array}{l}-20.1 \\
-\quad 1 \\
+\quad 3.5\end{array}$ & $\begin{array}{l}-19.2 \\
-\quad 1 \\
+\quad 3.3\end{array}$ & $\begin{array}{l}-18.3 \\
-\quad 1 \\
+\quad 31\end{array}$ & $\begin{array}{l}-17.4 \\
-\quad 1 \\
+\quad 29\end{array}$ & $\begin{array}{l}-16.6 \\
-\quad 1 \\
+\quad 2.7\end{array}$ & $\begin{array}{l}-149 \\
-\quad 1 \\
+\quad 23\end{array}$ & $\begin{array}{l}-13.1 \\
-\quad 1 \\
+\quad 19\end{array}$ & $\begin{array}{l}-113 \\
-1 \\
+\quad 15\end{array}$ \\
\hline $10 \mathrm{~cm}$. & $\begin{array}{l}10 \mathrm{~cm} . \\
20 \\
25\end{array}$ & $\begin{array}{l}-28.8 \\
-\quad 6 \\
-\quad 0.6\end{array}$ & $\begin{array}{l}-27.9 \\
-\quad 6 \\
-\quad 0.8\end{array}$ & $\begin{array}{r}-27 \\
-\quad 6 \\
-\quad 1\end{array}$ & $\begin{array}{l}-26.1 \\
-\quad 6 \\
-\quad 12\end{array}$ & $\begin{array}{l}-25.3 \\
-\quad 6 \\
-\quad 1.4\end{array}$ & $\begin{array}{c}-23.5 \\
-\quad 6 \\
-18\end{array}$ & $\begin{array}{l}-21.7 \\
-\quad 6 \\
-\quad 2.2\end{array}$ & $\begin{array}{l}-19.9 \\
-\quad 6 \\
-\quad 26\end{array}$ \\
\hline $11 \mathrm{~cm}$. & $\begin{array}{l}10 \mathrm{~cm} . \\
20 \\
25\end{array}$ & $\begin{array}{l}-37.6 \\
-11 \\
-\quad 4.8\end{array}$ & $\begin{array}{l}-36.7 \\
-11 \\
-\quad 5\end{array}$ & $\begin{array}{l}-35.8 \\
-11 \\
-\quad 5.2\end{array}$ & $\begin{array}{l}-34^{\circ} 9 \\
-11 \\
-\quad 5.4\end{array}$ & $\begin{array}{l}-34^{\circ} 1 \\
-11 \\
-\quad 5.6\end{array}$ & $\begin{array}{l}-32 \cdot 3 \\
-11 \\
-\quad 6\end{array}$ & $\begin{array}{l}-30.5 \\
-11 \\
-\quad 6.4\end{array}$ & $\begin{array}{r}28.7 \\
-\quad 11 \\
-\quad 6.9\end{array}$ \\
\hline
\end{tabular}


TABLE IV

\begin{tabular}{|c|c|c|c|c|c|c|c|c|c|}
\hline \multicolumn{2}{|c|}{ Stereogram } & \multicolumn{8}{|c|}{ Interocular Distance (cm.) } \\
\hline Width & $\mid \begin{array}{c}\text { Distance } \\
\text { from Lens }\end{array}$ & 46 & 48 & $5 \%$ & $5 \cdot 2$ & 54 & 5.8 & $6 \cdot 2$ & 6.6 \\
\hline $3 \mathrm{~cm}$. & $\begin{array}{l}11 \mathrm{~cm} . \\
25 \\
33: 3\end{array}$ & $\begin{array}{l}+27.6 \\
+24 \\
+23.1 \\
\end{array}$ & $\begin{array}{l}28 \cdot 5 \\
24 \\
22 \cdot 9\end{array}$ & $\begin{array}{l}29.4 \\
24 \\
227\end{array}$ & $\begin{array}{l}30 \cdot 3 \\
24 \\
22 \cdot 5\end{array}$ & $\begin{array}{l}31 \cdot 1 \\
24 \\
22 \cdot 3\end{array}$ & $\begin{array}{l}32 \cdot 9 \\
24 \\
21 \cdot 9\end{array}$ & $\begin{array}{l}34 \cdot 7 \\
24 \\
21 \cdot 5\end{array}$ & $\begin{array}{l}36 \cdot 4 \\
24 \\
21 \cdot 1\end{array}$ \\
\hline $4 . \mathrm{cm}$. & $\begin{array}{l}11 \mathrm{~cm} . \\
25 \\
33 \cdot 3\end{array}$ & $\begin{array}{l}+19 \cdot 8 \\
+20 \\
+20\end{array}$ & $\begin{array}{l}20.7 \\
20 \\
19 \cdot 8 \\
\end{array}$ & $\begin{array}{l}21 \cdot 6 \\
20 \\
19 \cdot 6\end{array}$ & $\begin{array}{l}22 \cdot 5 \\
20 \\
19 \cdot 4\end{array}$ & $\begin{array}{l}23 \cdot 3 \\
20 \\
19 \cdot 2\end{array}$ & $\begin{array}{l}25 \cdot 1 \\
20 \\
18.8\end{array}$ & $\begin{array}{l}26.9 \\
20 \\
18.4\end{array}$ & $\begin{array}{l}28 \cdot 6 \\
20 \\
18\end{array}$ \\
\hline $5 \mathrm{~cm}$. & $\begin{array}{l}11 \mathrm{~cm} . \\
25 \\
33.3\end{array}$ & $\begin{array}{l}+12 \\
+16 \\
+16.9\end{array}$ & $\begin{array}{l}12.9 \\
16 \\
16.7\end{array}$ & $\begin{array}{l}13.8 \\
16 \\
16.5\end{array}$ & $\begin{array}{l}14 \cdot 7 \\
16 \\
16 \cdot 3\end{array}$ & $\begin{array}{l}15 \cdot 5 \\
16 \cdot \\
16 \cdot 1\end{array}$ & $\begin{array}{l}16 \cdot 3 \\
16 \\
15 \cdot 7 \\
\end{array}$ & $\begin{array}{l}17 \cdot 1 \\
16 \\
15 \cdot 3 \\
\end{array}$ & $\begin{array}{l}20.8 \\
16 \\
14.9\end{array}$ \\
\hline $6 \mathrm{~cm}$. & $\begin{array}{l}11 \mathrm{~cm} . \\
25 \\
33 \cdot 3\end{array}$ & $\begin{array}{l}+4.2 \\
+12 \\
+13.8\end{array}$ & $\begin{array}{c}5.1 \\
12 \\
13.6\end{array}$ & $\begin{array}{l}5.9 \\
12 \\
13.4\end{array}$ & $\begin{array}{c}6.8 \\
12 \\
13.2\end{array}$ & $\begin{array}{l}7 \cdot 7 \\
12 \\
13\end{array}$ & $\begin{array}{l}9 \cdot 4 \\
12 \\
12.6\end{array}$ & $\begin{array}{l}11 \cdot 2 \\
12 \\
12 \cdot 2\end{array}$ & $\begin{array}{l}13 \\
12 \\
11 \cdot 8 \\
\end{array}$ \\
\hline $7 \mathrm{~cm}$. & $\begin{array}{l}11 \mathrm{~cm} . \\
25 \\
33 \cdot 3\end{array}$ & $\begin{array}{l}-3.6 \\
+\quad 8 \\
+10.7\end{array}$ & $\begin{array}{l}-2.7 \\
+\quad 8 \\
+10.5\end{array}$ & $\begin{array}{ll}- & 1.9 \\
+ & 8 \\
+10.3\end{array}$ & $\begin{array}{l}-1 \\
+10 \\
+10.1\end{array}$ & $\begin{array}{l}-0.1 \\
+\quad 8 \\
+\quad 9.9\end{array}$ & $\begin{array}{l}+1.6 \\
+8 \\
+\quad 9.5\end{array}$ & $\begin{array}{l}+3.4 \\
+8 \\
+\quad 9.1\end{array}$ & $\begin{array}{l}+5.2 \\
+8 \\
+\quad 8.7\end{array}$ \\
\hline $8 \mathrm{~cm}$. & $\begin{array}{l}11 \mathrm{~cm} . \\
25 \\
33 \cdot 3\end{array}$ & $\begin{array}{l}-11.4 \\
+\quad 4 \\
+\quad 7.6\end{array}$ & $\begin{array}{l}-10.5 \\
+\quad 4 \\
+\quad 7.4\end{array}$ & $\begin{array}{l}-9.6 \\
+\quad 8 \\
+7 \cdot 2\end{array}$ & $\begin{array}{l}-8 \cdot 8 \\
+4 \\
+\quad 7\end{array}$ & $\begin{array}{l}-7.9 \\
+4 \\
+\quad 6.8\end{array}$ & $\begin{array}{l}-6.2 \\
+\quad 4 \\
+\quad 6.4\end{array}$ & $\begin{array}{l}-4.4 \\
+4 \\
+\quad 6\end{array}$ & $\begin{array}{l}\bar{t} 2.6 \\
+\quad 4.6\end{array}$ \\
\hline $9 \mathrm{~cm}$. & $\begin{array}{l}11 \mathrm{~cm} . \\
25 \\
33 \cdot 3\end{array}$ & $\begin{array}{r}-19.2 \\
+\quad 4.5\end{array}$ & $\begin{array}{r}-18.3 \\
+\quad 0 \\
+\quad 4.3\end{array}$ & $\begin{array}{c}-17 \cdot 4 \\
0 \\
+\quad 4 \cdot 1\end{array}$ & $\begin{array}{c}-16.6 \\
0 \\
+\quad 3.9\end{array}$ & $\begin{array}{r}-15.7 \\
+\quad 0 \\
+\quad 3.7\end{array}$ & $\begin{array}{r}-14 \\
+\quad 0 \\
+\quad 3.3\end{array}$ & $\begin{array}{r}-12 \cdot 2 \\
+\quad 0 \\
+\quad 2.9\end{array}$ & $\begin{array}{c}-10.4 \\
+\quad 0.5 \\
+\quad 2.5\end{array}$ \\
\hline $10 \mathrm{~cm}$. & $\begin{array}{l}11 \mathrm{~cm} . \\
25 \\
33 \cdot 3\end{array}$ & $\begin{array}{r}-27 \\
-\quad 4 \\
+\quad 1.4\end{array}$ & $\begin{array}{c}-26 \cdot 1 \\
+4 \\
+\quad 1 \cdot 2\end{array}$ & $\begin{array}{l}-25 \cdot 2 \\
\bar{T} \\
+\quad 1\end{array}$ & $\begin{array}{cc}-24.4 \\
-4 \\
+\quad 0.8\end{array}$ & $\begin{array}{l}-23.5 \\
\overline{1} \quad 4 \\
0.6\end{array}$ & $\begin{array}{l}-21 \cdot 8 \\
-\quad 4 \\
+\quad 0.2\end{array}$ & $\begin{array}{l}-20 \\
=\quad 4 \\
-\quad 0.2\end{array}$ & $\begin{array}{l}-18.3 \\
=4 \\
=0.6\end{array}$ \\
\hline $11 \mathrm{~cm}$. & $\begin{array}{l}11 \mathrm{~cm} . \\
25 \\
33 \cdot 3\end{array}$ & $\begin{array}{l}-34.8 \\
=8 \\
=\quad 1.7\end{array}$ & $\begin{array}{r}-33.9 \\
8 \\
1.9\end{array}$ & \begin{tabular}{c|}
33 \\
8 \\
$2 \cdot 1$
\end{tabular} & $\begin{array}{c}32 \cdot 3 \\
8 \\
2 \cdot 3\end{array}$ & $\begin{array}{c}31 \cdot 3 \\
8 \\
2 \cdot 5\end{array}$ & $\begin{array}{c}29 \cdot 6 \\
8 \\
2 \cdot 6\end{array}$ & $\begin{array}{c}27 \cdot 8 \\
8 \\
3 \cdot 3\end{array}$ & $\begin{array}{c}26.1 \\
8 \\
3 \cdot 7\end{array}$ \\
\hline
\end{tabular}

If, however, accommodation is also to be varied, the simpler stereoscope is more effective-again provided that one is fully aware of how it should be used; and this brings one to the interesting consideration of the relationship, when using a stereoscope, between accommodation and convergence. In this connection a Table has been calculated showing the position of any card at which accommodation: balances convergence. The range over which this can obtain is limited. Cards separated by a distance of less than $4 \mathrm{cms}$. cannot be brought sufficiently close to the lenses to induce the required amount of accommodation. On the other hand, beyond the focal plane accommodation is absent 
or negative, and cards separated by more than $10 \mathrm{cms}$. require divergence in all positions for superimposition to occur. Within the remaining range, however, the convergence can be calculated from the figures in Tables III and IV and expressed in metreangles by dividing the figure representing prism dioptres of convergence by the interocular distance in cms. Thus, taking the first item in Table III, $\frac{32 \cdot 2}{4.6}=7$ M.A. The results are shown in Table $\mathrm{V}$, and are of interest in demonstrating the wide fluctuations in the accommodation factor induced by movement of the stereograms. The Table enables one to apply an exercise for

TABLE V

\begin{tabular}{l|c|c|c|c}
\hline \multirow{2}{*}{$\begin{array}{c}\text { Stereogram } \\
\text { Width }\end{array}$} & \multicolumn{2}{|c|}{ 5 OD. LENSES } & \multicolumn{2}{|c}{4 OD. LENSES } \\
\cline { 2 - 4 } & $\begin{array}{c}\text { Distance } \\
\text { from } \\
\text { Lens }\end{array}$ & Acc (D) = Conv (M.A.) & $\begin{array}{c}\text { Distance } \\
\text { from } \\
\text { Lens }\end{array}$ & Acc (D) = Conv (M.A.) \\
\hline 4 cms. & 9.5 & 4.5 & 11.7 & 4.4 \\
$5 \mathrm{cms}$. & 11.4 & 3.4 & 15 & 2.9 \\
$6 \mathrm{cms}$. & 13.8 & 22 & 18 & 1.9 \\
$7 \mathrm{cms}$. & 16.4 & 1.2 & 20.2 & 1.1 \\
$8 \mathrm{cms}$. & 18.5 & 0.5 & 22.8 & 05 \\
$9 \mathrm{cms}$. & 20 & 0 & 25 & 0 \\
\hline
\end{tabular}

improving accommodation and convergence together with scientific accuracy; the bar of the stereoscope could with advantage be marked to indicate the accommodative effort demanded.

It is a pleasure to express my grateful thanks for the courteous and enthusiastic collaboration of $\mathrm{Mr}$. S. 'P. Holloway in the preparation of this article, and for his drawing up of the Tables. 